\title{
Novel Signal-Enhancing Approaches for Optical Detection of Nucleic Acids-Going beyond Target Amplification
}

\section{Laura Miotke $^{1}$, Maria Carla Barducci ${ }^{2}$ and Kira Astakhova ${ }^{2, *}$}

1 Division of Oncology, Department of Medicine, Stanford University School of Medicine, Stanford, CA 94305, USA; E-Mail: 1miotke@gmail.com

2 Nucleic Acid Center, Department of Physics, Chemistry and Pharmacy, Campusvej 55, Odense M 5230, Denmark; E-Mail: mabar14@student.sdu.dk

* Author to whom correspondence should be addressed; E-Mail: ias@sdu.dk;

Tel.: +45-6550-25-23; Fax: +45-6615-87-80.

\section{Academic Editor: Igor Medintz}

Received: 10 August 2015 / Accepted: 16 September 2015 / Published: 23 September 2015

\begin{abstract}
Detection of low-abundance nucleic acids is a challenging task, which over the last two decades has been solved using enzymatic target amplification. Enzymatic synthesis enhances the signal so that diverse, scientifically and clinically relevant molecules can be identified and studied, including cancer DNA, viral nucleic acids, and regulatory RNAs. However, using enzymes increases the detection time and cost, not to mention the high risk of mistakes with amplification and data alignment. These limitations have stimulated a growing interest in enzyme-free methods within researchers and industry. In this review we discuss recent advances in signal-enhancing approaches aimed at nucleic acid diagnostics that do not require target amplification. Regardless of enzyme usage, signal enhancement is crucial for the reliable detection of nucleic acids at low concentrations. We pay special attention to novel nanomaterials, fluorescence microscopy, and technical advances in detectors for optical assessment. We summarize sensitivity parameters of the currently available assays and devices which makes this review relevant to the broad spectrum of researchers working in fields from biophysics, to engineering, to synthetic biology and bioorganic chemistry.
\end{abstract}

Keywords: enzyme-free detection; low-abundance nucleic acids; detection by optical methods; Specificity; Sensitivity; SNP/SNV analysis 


\section{Introduction}

Modern natural sciences and medicine actively apply nucleic acid detection in vitro, in cell culture and in vivo. For this, the number of nucleic acid targets per sample volume is a crucial parameter for reliable detection. It has become possible to control the target abundance in biological samples through assays like polymerase-chain reaction (PCR) and specific small interfering RNA (siRNA) knockdowns [1]. However, upon enzymatic manipulation there is a high risk of affecting the initial biomolecular system that underlies the biological function of the target molecule [2]. Therefore, there is a growing need to detect biomolecules at their native abundance levels, which is many orders of magnitude lower than when using amplification.

Nucleic acids are present in cells at the level of approximately $10^{1}-10^{8}$ copies per cell [3]. Furthermore, their distribution within the sample often varies over the course of the experiment and can be an actual objective of the study. In a recent paper we gave an overview of enzymatic strategies involving amplification of nucleic acid targets in comparison to rapidly developing enzyme-free assays [4]. As discussed in this paper, PCR-independent methods without additional signal enhancing strategies yielded limit of target detection (LOD) values of $5 \mathrm{nM}$, which is not suitable for most biological applications [3,4]. Signal-enhancing strategies are therefore envisioned as a necessary tool for reliable, sensitive detection in enzyme-free assays.

In this review, we describe recent developments in signal-enhancing strategies that can be used for the detection of low-abundance nucleic acids by fully enzymatic-, partially enzymatic- and completely enzyme-free assays. We dedicate the main focus of this paper to the novel tools facilitating highly sensitive optical detection strategies including colorimetry, fluorometry, fluorescence microscopy, and surface plasmon resonance (SPR). We divide these tools into three groups: (1) enzyme-labeling strategies which do not amplify target nucleic acid; (2) enzyme-free hybridization platforms; (3) macrofluorophores and nanoparticles. In addition, we review the advances in label-free detection using SPR and compare a handful of optical techniques in terms of the sensitivity of their detector devices.

\section{Enzyme Labeling Strategies without Polymerase Target Amplification}

In enzyme labeling, the target sequence is detected by a target-specific reagent (e.g., synthetic oligonucleotide probe) linked to an enzyme either by a covalent bond or through a secondary complex. Major applications of enzyme labeling include enzyme-linked immunosorbent assays (ELISAs), immunocytochemical and immunohistochemical analyses, and nucleic acid hybridization assays. Protein enzymes are applied in ELISA, immunocytochemical and immunohistochemical analyses, whereas DNA manipulating enzymes find applications in nucleic acid hybridization assays [5,6].

In recent years, enzyme-labeling techniques have been able to provide the same sensitivity as PCR without the need for target amplification. One particular technique, the Simoa assay, demonstrates a LOD of approximately $70 \mathrm{aM}$ [6]. The principle of Simoa is to bind the target DNA to magnetic particles by using specific capture DNA sequences. Then, a second pool of probes containing biotin labels is then used to detect the target sequences. The hybridized biotin-labeled probes bind streptavidin-conjugated enzyme $\beta$-galactosidase, and the $\beta$-galactosidase converts a colorless fluorescein substrate into the purple oxidized derivative detected by fluorometry [6] (Figure 1A). 
Importantly, all the steps in the Simoa assay are fully automated and allow for the rapid multiplexed detection of nucleic acids and proteins directly in biological samples.

A.

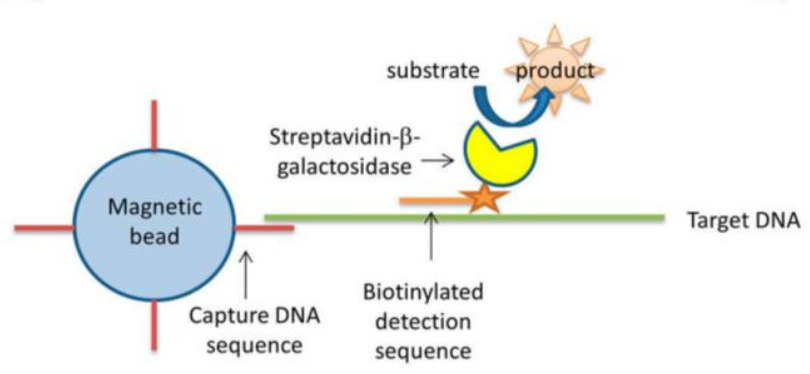

B.

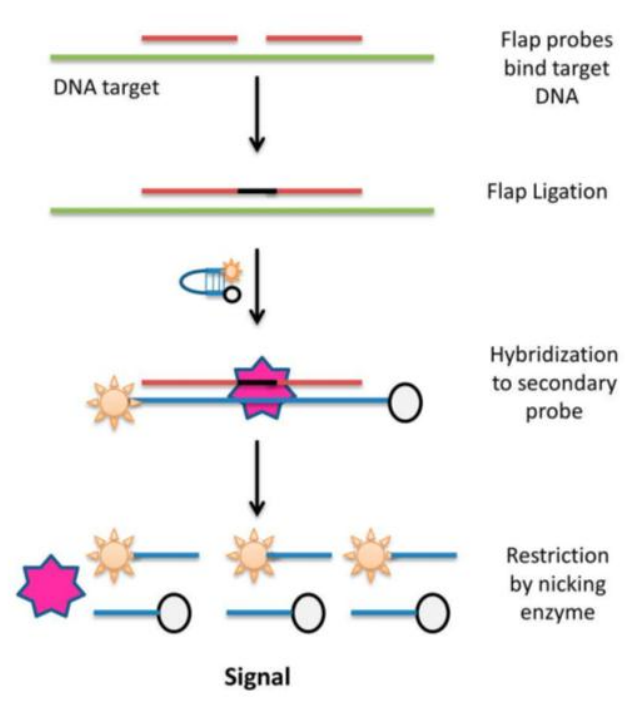

Figure 1. General principles of Simoa (A) and flap endonuclease based (B) assays.

The protein enzyme frequently applied in ELISA and similar assays is horseradish peroxidase (HRP). This enzyme converts colorless organic substrate 3,3,5,5-tetramethylbenzidine (TMB) into the colored oxTMB in presence of catalytic amounts $\mathrm{H}_{2} \mathrm{O}_{2}$. For nucleic acid detection, ELISA can be performed either directly using HRP-conjugated antibody, or indirectly, when a secondary anti-antibody is applied [5]. In Step one, the sample, which potentially contains a target molecule, is applied to a pre-coated surface. After a wash step, the surface is subjected to binding with a secondary antibody labeled with HRP (Step 2). Given that the target molecule is present in the analyte, Step 2 will result in the binding of the corresponding number of HRP molecules to the surface. Finally, TMB is added and the absorbance measurement at the characteristic wavelength for oxTMB (450 nm), revealing the amount of target in the initial sample.

DNA-manipulating enzymes for nucleic acid diagnostics are exemplified by thermostable ligases. These enzymes can be used in combination with DNA specific hybridization probes and provide a so-called "ligase chain reaction" when the target DNA is bound. The signal from the DNA target is boosted without the use of a polymerase, however the target is still being enzymatically amplified. This technique allows for detailed analysis of single-nucleotide polymorphisms (SNPs) [7].

DNAzymes are catalytically active DNA molecules that exhibit different enzymatic activities based on their sequence and structure. For example, the peroxidase-like activity of G-quadruplex based DNAzymes was used in several reports for detection of nucleic acids. In one, DNAzyme molecules with peroxidase-like activity were incorporated into target specific oligonucleotides that were then used to monitored nucleic acids in colorimetric or chemiluminescent assays [8]. In other applications, they were used for the quantification of metal cations and to amplify detection of small molecules and proteins ranging from adenosine, cocaine, or AMP, to lysozyme and thrombin [8-10].

Flap endonucleases are another example of DNA-manipulating enzymes for signal-enhancement [11]. As demonstrated in Figure 1B, these assays utilize sequence-specific cleavage of nucleic acid structures in target-specific probes. A target-probe binding event results in the displacement of these 
nucleic acid structures by a second pool of DNA containing restriction sites for endonucleases. This in turn results in a cleavage-generated nucleic acid fragment, which becomes a substrate for secondary reactions. These secondary reactions exponentially generate FRET probes giving off a detectable signal [11].

Notably, some inorganic materials can also have catalytic activity that is applicable in nucleic acid detection. For example, Song and co-workers applied intrinsic peroxidase-like activity of single-walled carbon nanotubes (SWNTs) to detect nucleic acid targets with a simple colorimetry [12]. Similarly to the HRP enzyme described above, SWNTs catalyze the reaction of the peroxidase substrate (TMB) giving the blue oxidized TMB product (oxTMB) when $\mathrm{H}_{2} \mathrm{O}_{2}$ is present. Using this approach, single-stranded (ss) DNA targets were detected in a complex with SWNTs in just 15 min at $1 \mathrm{nM}$ concentration of DNA (12).

Additional signal-enhancing effects can be achieved by binding multiple enzymes per each target molecule. In recent work, hybridization chain reaction amplification of microRNA using DNA nanostructure based sensors allowed for target DNA detection at an extraordinarily low LOD of 600 copies $/ 100 \mu \mathrm{L}$ of sample [13]. A chain reaction of recognition and hybridization events between two sets of DNA hairpin molecules offered the rapid detection of specific DNA sequences. In addition each hairpin contained a conjugated enzyme, which resulted in a dramatic signal-enhancing effect upon the attachment to an immobilized target:capture complex [13].

\section{Enzyme-Free Hybridization Assays: Specificity in Focus}

Highly specific and sensitive hybridization probes enable the detection of target nucleic acids without the need for additional enzymes and at previously unachievable single-molecule resolution [14]. Unlike PCR, such probes amplify only the signal and not the target, and unlike enzyme-linked reagents they only involve restriction enzymes. Over the past couple decades, hybridization probes have become a fundamental aspect of widely applied techniques such as fluorescence in situ hybridization (FISH), flow cytometry, and fluorescence microscopy [14-16]. Among other approaches for enzyme-free signal-enhancement we distinguish two major strategies: branched DNA (bDNA) or sequence amplification through cascade hybridization, and signal-enhancing by laser microscopy. Additionally, the strategies described below can be combined with enzymatic assays to further increase sensitivity.

Branched DNA probes have been around for over 20 years and still find multiple applications in clinical research and biotechnology. bDNA probes contain a 20-25 nucleotide (nt) region which specifically binds the target, a short linker region, and a binding site for multiple sequences containing fluorescent dyes or enzymes (Figure 2). In this way, the target-specific signal is increased enough to perform enzyme-free mRNA sensing at LOD of only 80 target copies/mL [17-19]. Importantly, bDNA assay is then less spending time consuming PCR methods with a complete turnaround of one day including DNA extraction [17-19]. However, ultrasensitive bDNA assays have to be used with caution on low target abundance samples since false positive results can occur [19]. This point has been demonstrated in the diagnosis of HIV-1 RNA viral load measurements in perinatally exposed infants. Samples with $>1800$ copies/mL had a good correlation with their retrospective picture whereas $<440$ copies/mL detected by bDNA gave multiple false positive results [19]. 


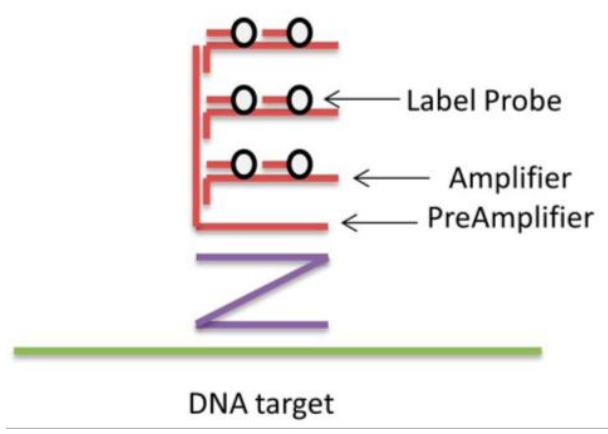

Figure 2. General principle of branched DNA (bDNA) assay.

In terms of signal visualization, powerful optical methods, such as fluorescence microscopy, increase the sensitivity of detection by several orders of magnitude $[15,16]$. In recent years, fluorescence microscopy has been gaining growing attention for detection of low abundance biomolecules, in particular for mRNA imaging experiments. So far this has been limited exclusively to research applications [20,21]. Device simplification and accessible data processing will further enhance the broad application of fluorescence microscopy in enzyme-free nucleic acid detection [22,23].

In hybridization assays at the single molecule level, probe specificity becomes a crucial parameter to ensure low off-target signals. According to recent research, probe specificity can be dramatically improved using synthetic nucleic acid analogues such as locked nucleic acids oxo-LNA (L) and functionalized 2'-amino-LNA analogues, Figure 3A; [4]. The structural and thermodynamic parameters of LNA/DNA oligonucleotides are different compared to non-modified DNA. For example, they have a higher resistance to enzymatic degradation and a higher binding affinity and selectivity to DNA and especially RNA targets. Moreover, LNA molecules can be combined with fluorescent markers in the form of either fluorogenic probes or dyes that can be directly attached to the 2'-amino position of 2'-amino-LNA (Figure 3). Short fluorescent LNA/DNA probes containing bright photostable polyaromatic hydrocarbons (PAHs) such as pyrene, (phenylethynyl)pyrenes and perylene represent a class of probes with the advantage of high target specificity and sensitivity [4]. Without target amplification, genotyping of HIV-1 protease encoding gene fragment (HIV PR) was performed with a pyrene probe yielding a LOD below $5 \mathrm{nM}$ [24]. Using (phenylethynyl)pyrene dyes, which have improved photophysical characteristics compared to the parent pyrene, the detection limit dropped even further below $5 \mathrm{nM}$. Importantly, this was achieved with an extraordinarily high LNA/DNA probe specificity and bright fluorescence response to a particular mismatched nucleotide. This presents an opportunity for using this technique to detect SNPs and SNVs.

Perylene-LNA/DNA probes have additional applications in the detection of RNA by fluorescence microscopy in cell cultures (Figure 3A,B) [25]. Short oligonucleotide probes containing perylene-LNA modification $\mathbf{2}$ and additional oxo-LNAs 1 specifically revealed the presence of target mRNA in fixed cells at excitation/emission wavelengths which completely excluded the cell's autofluorescence (Figure 3). More recently, we developed new "clickable" probes for fluorescence microscopy of biologically active circular RNA and microRNA (Figure 3C) [26]. These new probes have the advantage of high photostability and bright fluorescence at the excitation/emission wavelengths suitable for in vivo assays. In addition, as with the (phenylethynyl)pyrene probes described above, these probes have strong sensitivity to SNPs in the target RNAs. To harness this characteristic further, 
we synthesized "clickable" 2'-amino-LNA derivatives for direct attachment of fluorophores to the $2^{\prime}$-amino-position via a 1,2,3-triazole linker (Figure 3D) [27]. As we demonstrate in a recent paper [27], attaching the fluorophores directly to the fluorescently labelled 2'-amino-LNA nucleotides improves the SNP discrimination of DNA and RNA targets. This makes them a reliable tool for allele-specific detection of nucleic acids by fluorescence microscopy. Moreover, 2'-alkyne functionality can be attached to diverse fluorophores and nanoparticles under highly efficient azide-alkyne cycloaddition conditions. Attachment of ultra-bright BODIPY dyes and semiconductor nanocrystals called quantum dots (QDs) is especially promising for applications in fluorescent microscopy (Figure 3D) [28,29]. In addition, fluorescent metal nanoclusters (for example, silver- or gold-based) have attractive optical qualities, which may increase the sensitivity of nucleic acid detection [30,31]. Remarkably, metal nanoclusters can detect natural nucleic acids, such as cancer related mRNAs, directly in living cells [31].

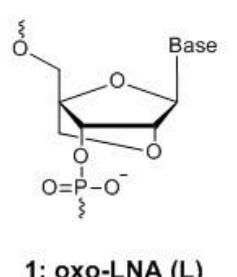

1: oxo-LNA (L)
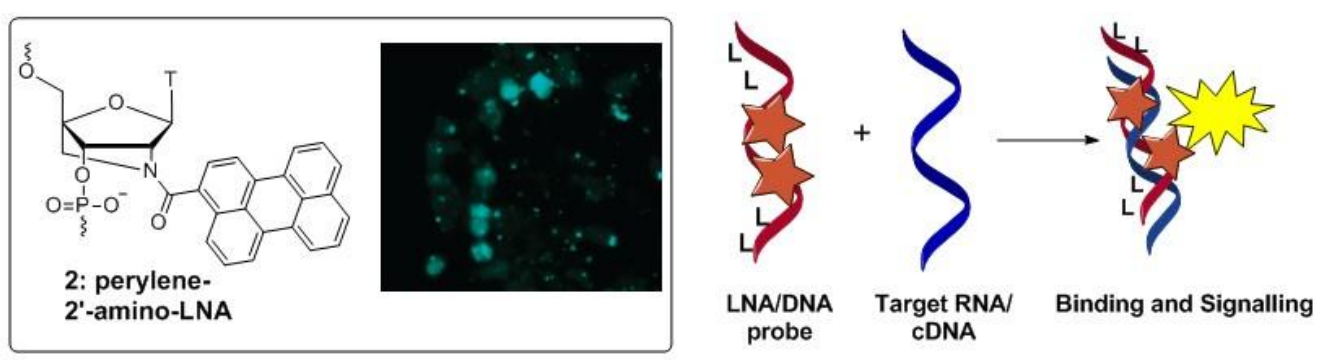

CDNA

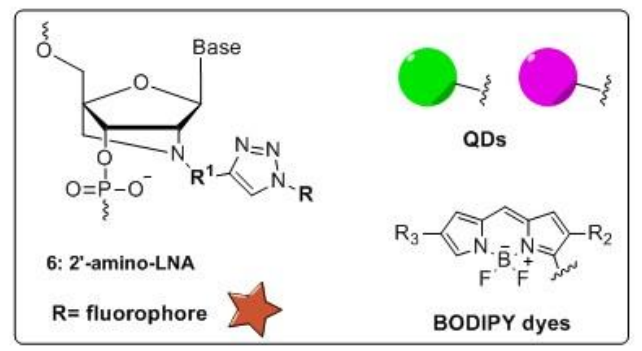

Figure 3. (A) Structure of oxo-LNA (L), perylene- 2 -amino-LNA and mRNA detection using fluorescence microscopy and perylene-2'-amino-LNA labeled oligonucleotide probe; (B) Principle of target signaling by fluorescent LNA/DNA probes;

(C) New 1,2,3-triazole-uridne nucleotide derivatives for fluorescence microscopy;

(D) General structures of 1,2,3-triazole-amino-LNA nucleotides; Quantum Dots (QDs) and BODIPY dyes.

Finally, the use of so-called environmentally sensitive modified nucleosides and nucleotides also offers exciting opportunities for the specific detection of nucleic acids [32-36]. The distinguishing feature of these is enhanced fluorescence upon a change in the local microenvironment. Thus, environmentally sensitive nucleosides signal the presence of a target by changing optical properties. This is typically achieved with diverse photochemical mechanisms and structural transitions. For example, in a recent study, nucleosides covalently linked to viscosity-sensitive fluorescent molecular rotors were used to light-up in the presence of target proteins [32]. In another paper, a naphthylethynylated adenosine derivative was used within synthetic probes to detect SNPs. They were able to achieve this owing to the environmentally sensitive intramolecular charge transfer (ICT) 
between the nucleobase and naphthalene moieties [33]. Other successful examples of environmentally sensitive modified nucleosides include light-up probes for the detection of triplex formation and polarity sensitive probes [34-36]. One particularly useful mechanism involves introducing intramolecular conformational changes at the bond between the attached modifications and nucleobase [34,35]. This makes modified nucleosides attractive objects of study with a high potential in diagnostics and structural studies of biomolecules.

\section{Ultra-Bright Fluorophores for Nucleic Acid Labeling: Macrofluorophores, Nucleic Acid Binding Dyes, and Nanoparticles}

Ultra-bright fluorophores allow for detection at low target abundance and can be combined with microarray, bDNA, or microscopy assays. One promising approach to increasing fluorescence brightness is polymerization of a single fluorophore into a large polymer nanoparticle, or so-called macrofluorophore (Figure 4). Collections of fluorophores numbering in the tens to millions form a common scaffold (Life Technologies). The scaffold is then coupled to a target-specific affinity reagent such as streptavidin or an oligonucleotide. The incorporated fluorophores are thereby collectively associated with the target upon binding. In spite of extreme brightness, macrofluorophores have several challenges such as difficulty in (1) achieving monodispersity (i.e., homogeneity in molecular weight) of nanoparticles upon synthesis; (2) selective covalent attachment to probes; (3) cellular uptake of large nanostructures for studies in cells. According to the literature, the first point is addressed by using so-called seeded polymerization (Figure 4). In this approach a starting material is co-polymerized with an organic agent (e.g., styrene) into small "seed" nanoparticles, which are then re-polymerized and stabilized into final products [37]. In this way, mono-dispersed macro-porous particles, approximately $10 \mu \mathrm{m}$ in size, were obtained. The second disadvantage of macrofluorophores is countered by covalently attaching them to oligonucleotides with NHS, disulfide, or click chemistry [38]. To address the third point, large nanomaterials can be delivered into cells using transfection reagents [38]. As an example latex macrofluorophores, available from LifeTechnologies, have been documented by imaging techniques to be taken up into the cell [39]. Ihara, et al., and Thomson, et al., employed these techniques recently to demonstrate successful examples of signal enhancement using fluorescent organic polymers with a p53 SNP sensitivity assay for DNA detection and an HSV DNA detection at sub-picomolar levels, respectively [40,41].

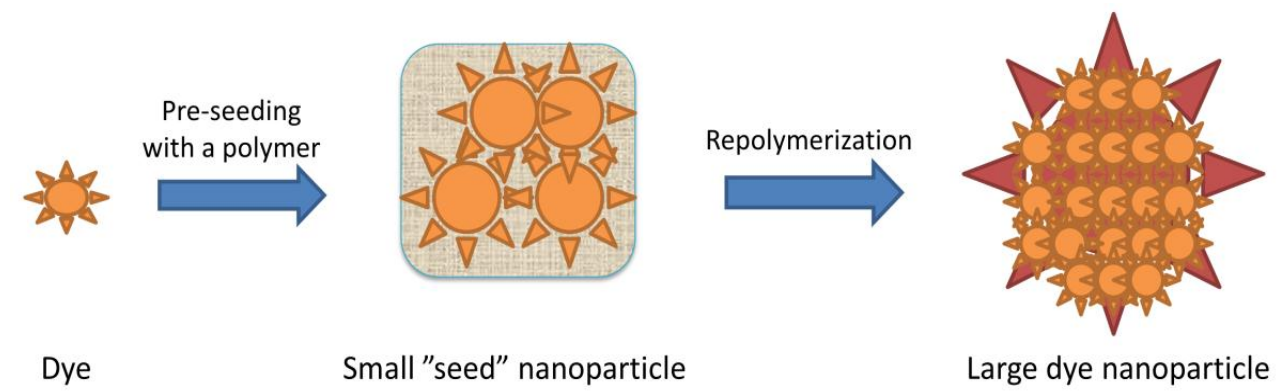

Figure 4. Seeded polymerization for the preparation of fluorescent nanoparticles.

An alternative approach to signal enhancement using fluorophores is the use of nucleic acid intercalating and groove binding dyes $[42,43]$. These types of molecules have been applied over the 
past 20 years in enzyme-based techniques, such as RT-qPCR [42,43]. Over the course of this period, highly toxic intercalators, such as ethidium bromide, have been replaced with less harmful molecules such as SybrGreen, EvaGreen, and QuantiFluor [42-44]. Briefly, the nucleic acid binding dyes have a quenched fluorescence in the unbound state in aqueous medium and an increased signal when the dye interacts with the nucleic acid sequence. Notably, these organic dyes have a high potential for quantification of large genomic fragments without needing amplification. This is owed to the fact that fluorescence is proportionally increased with an increasing number of base pairs of DNA. Indeed this principle is the basis for concentration determination assays for ssDNA and dsDNA such as those developed by Promega [44]. Since interaction shows little to no sequence specificity, however, applications of intercalating and groove binding dyes are so far limited to in vitro studies.

Lastly, nanoparticles present an additional avenue for boosting the fluorescence signal in nucleic acid labels. One approach is preparing target-specific probes containing ultra-bright fluorescent materials with fluorescent carbon nanoparticles (FCNs) and quantum dot nanocrystals (QDs) [38,45-47]. FCNs are nano-crystalline material, with predominantly graphitic structure, that fluoresce upon UV light exposure. It has been shown that FCNs have promising characteristics in sensing nucleic acids, given that their effective synthesis and increased quantum yields are achieved [45]. The main advantage of FCNs compared to other nanomaterials is their low toxicity, which allows for their application in environment friendly diagnostics and gene therapy [45,46]. In particular, recently reported water-soluble FCNs were used successfully in cell imaging probes that sensitively detected natural nucleic acids [47]. From a physical perspective, QDs are single fluorophores with extraordinary photon output capacity. However, in terms of optical properties QDs resemble macrofluorophores and are similar in size to the smallest fluorescent microspheres. QDs have been used successfully in nucleic acid assays in cells and single-molecule imaging studies in vivo [38].

Complexes between DNA and negatively charged gold nanoparticles (Au-nps) have been studied for many years and have recently been found useful in specific nucleic acid detection with colorimetry [4,38]. These assays utilize the dispersion and aggregation of the Au-nps, either with a cross-linking or non-cross-linking aggregation mechanism [48]. For cross-linking aggregation, several interparticular DNA molecules accelerate Au-np aggregation. In turn, non-cross-linking aggregation uses changes in electrostatic or (electro) steric stabilization of the Au-np surface. This is suitable for detection of ssDNA, for example [49]. In a recent paper, Au-nps were incorporated into a hybridization chain reaction assay for SNP detection in model DNA targets. Remarkably, they achieved a LOD of $50 \mathrm{pM}$ with instrument detection and $100 \mathrm{pM}$ with naked eye observation, which is about two orders of magnitude lower than that of previously reported Au-np-based assays [50].

\section{Signal Enhancement in Surface Plasmon Resonance Assays}

Surface plasmon resonance (SPR) spectroscopy is an optical surface analysis technique that measures refractive index changes induced by molecular adsorption on a metal film. In general, SPR is based on the resonant oscillation of electrons at the interface between a negative and positive permittivity material stimulated by incident light. The resonance condition is established when the frequency of incident photons matches the natural frequency of surface electrons oscillating against the restoring force of positive nuclei. The resonance conditions are unique and informative for a particular ligand-target interaction when one of them is immobilized on the SPR chip surface and the other is 
applied in the solution flowing through the SPR cell. Therefore SPR is the basis of many adsorption measurements applied in biosensors.

Biacore launched the first SPR instrument in 1990. Since than multiple SPR-based detection systems have been developed and applied to nucleic acid detection [51-56]. SPR has the advantage of being a fast, fully automated, label-free method, which requires very small sample and reagent volumes. However, from its conception the inability of SPR to measure low abundance targets has limited its applications to PCR products and synthetic nucleic acids at high concentrations. In $2008 \mathrm{Su}$ and co-authors reported a signal-enhancing approach for SPR using HRP-assisted oxidative aniline polymerization. In addition, they used a DNA binding cationic polymer which affects the refractive index of DNA [51]. This decreased the LOD down to $0.1 \mathrm{pM}$ compared to the low $\mathrm{nM}$ range of previous assays. In more recent papers, other techniques were applied to SPR spectroscopy to increase the signal. For example, using a super-sandwich DNA-streptavidin technique [52] or biofunctionalized Au-nps [53] results in LODs in the low picomolar range.

The sensitivity of PCR-independent DNA detection by SPR could be further improved to 0.3-3 fM by using the previously mentioned hybridization chain reaction [54], DNAses [55] or graphene-coated SPR interfaces [56]. Graphene-coated SPR technique applied non-covalent functionalization of SPR chip which was pre-coated with graphene with gold nanocrystals carrying sequence-specific DNA capture probes. Graphene improved the adsorption of the capture probes through hydrogen bonding and $\pi$ - $\pi$-staking interactions. As a result, Au-nanocrystals containing ssDNA, with a higher mass than original ssDNA, could be attached. Upon hybridization with the complementary target, desorption of the nanocrystals occurred with a high change in a refractive index (approx. 20 times larger than ssDNA on a graphene SPR chip alone). This enabled for the detection of the DNA hybridization event at a target concentration of as low as $0.5 \mathrm{fM}$ [56].

\section{Signal Enhancement with Optical Detectors}

In optical assays the signal is received using a technical device called a detector that varies significantly between different optical techniques. It is crucial for the assay that the light source and the detector are selected correctly. The technical parameters of these devices put requirements on the probe design, assay conditions and chemistry and photochemistry of the applied fluorophores.

A summary of currently applied optical techniques is presented in Table 1. Among optical methods, colorimetry is the simplest and most accessible approach. It uses a portable apparatus (approx. $1 \mathrm{~kg}$ in weight) and is compatible with most labels and aforementioned signal-enhancing techniques. Using a simple photoresistor as a signal detector the LOD is $50 \mathrm{pM}$ of DNA [50]. However, colorimetry can only be applied when the probe's absorbance wavelengths lie within the visible region [57]. In turn, fluorometers allow for detection within a broad spectral region if their absorbance, fluorescence, and luminescence modes are utilized [57-61]. The applications of fluorometers are very diverse and they are applied in diagnostic assays such as ELISAs and RT-qPCR. Horiba spectrofluorometers especially have the advantage of high sensitivity and a broad wavelength range. Remarkably, these fluorometers are equipped with a photonmultiplier tube (PMT) that are also often applied in the microscopy [62,63]. This acts as a signal enhancer for the main detector in most fluorometry-based techniques applied to date. PMTs contain a photocathode, several dynodes, and an anode. 
Table 1. Specifications of optical methods used for amplification-free, low-abundance, nucleic acid detection.

\begin{tabular}{|c|c|c|c|c|c|c|}
\hline \multirow[b]{2}{*}{ Assay/Producer } & \multicolumn{2}{|c|}{ Apparatus Specifications: } & \multirow{2}{*}{$\begin{array}{c}\text { Compatible Labels; } \\
\text { Absorbance-/Emission } \\
\text { (abs-em) Range, nm; QY }\end{array}$} & \multicolumn{3}{|c|}{ LOD * } \\
\hline & $\begin{array}{l}\text { Light } \\
\text { Source }\end{array}$ & Detector & & Application & pmol & Ref. \\
\hline $\begin{array}{c}\text { Colorimetry: Konica } \\
\text { Minolta/Hach }\end{array}$ & $\begin{array}{c}\text { Filament } \\
\text { lamp or LED }\end{array}$ & PR & $\begin{array}{l}\text { Chromophore with abs at } \\
\quad 380-780 \mathrm{~nm}\end{array}$ & DNA & $5 \times 10^{-3}$ & {$[50,57]$} \\
\hline $\begin{array}{c}\text { Fluorometry: } \\
\text { Promega Glomax }\end{array}$ & $\begin{array}{l}\text { Xe lamp or } \\
\text { LED }\end{array}$ & $\mathrm{PD} / \mathrm{EPC}+\mathrm{PMT}$ & $\begin{array}{l}\text { Fluorophore with abs-em at } \\
260-750 \mathrm{~nm}, \mathrm{QY}>0.1\end{array}$ & Luciferase & $3 \times 10^{-9}$ & {$[58]$} \\
\hline $\begin{array}{c}\text { Horiba } \\
\text { spectrofluorometer }\end{array}$ & Xe lamp & $\mathrm{PD} / \mathrm{EPC}+\mathrm{PMT}$ & $\begin{array}{c}\text { Fluorophore with abs-em at } \\
185-1700 \mathrm{~nm}, \mathrm{QY}>0.1\end{array}$ & DNA & $\begin{array}{l}\text { FLS: } 9 \times 10^{-4} \\
\text { LSC: } 2 \times 10^{-4}\end{array}$ & {$[59]$} \\
\hline $\begin{array}{l}\text { TECAN multimode } \\
\text { reader: Infinite F200 } \\
\text { PRO } \\
\end{array}$ & Xe lamp & $\mathrm{PD} / \mathrm{EPC}+\mathrm{PMT}$ & $\begin{array}{l}\text { Fluorophore with abs-em at } \\
230-1000 \mathrm{~nm}, \mathrm{QY}>0.1\end{array}$ & DNA & $\begin{array}{c}\text { FLS: } 3 \times 10^{-4} \\
\text { LSC: } 1.2 \times 10^{-5}\end{array}$ & {$[60]$} \\
\hline $\begin{array}{l}\text { RT-qPCR: Roche } \\
\text { Light Cycler } 480\end{array}$ & HI LED & $\mathrm{CCD}$ & $\begin{array}{c}\text { Fluorophore with abs-em at } \\
488-660 \mathrm{~nm}, \mathrm{QY}>0.1\end{array}$ & DNA & $3 \times 10^{-3}$ & {$[61]$} \\
\hline $\begin{array}{c}\text { Microscopy: } \\
\text { Nikon/Leica/Olympu } \\
\text { s/Zeiss/Horiba }\end{array}$ & $\begin{array}{l}\text { Lamp (Xe, } \\
\mathrm{MH}) / \mathrm{LED}\end{array}$ & $\begin{array}{c}\mathrm{PD} / \mathrm{EPC}+\mathrm{PMT} \\
\mathrm{CCD}, \mathrm{CID} \\
\mathrm{CMOS}, \\
\mathrm{EBCCD} ; \mathrm{GaAsP}\end{array}$ & $\begin{array}{c}\text { Photostable fluorophores with } \\
\text { abs-em at } 240-940 \mathrm{~nm}, \\
\text { optimal at QY > } 0.6\end{array}$ & DNA/RNA & $<1 \times 10^{-13}$ & {$[62]$} \\
\hline $\begin{array}{l}\text { Surface plasmon } \\
\text { resonance: Biacore, } \\
\text { BioNavis }\end{array}$ & $\begin{array}{l}\text { Lamp, diode, } \\
\text { laser or fiber }\end{array}$ & $\mathrm{PD}$ or $\mathrm{CCD}$ & $\mathrm{n} / \mathrm{a}$ & DNA & $3 \times 10^{-4}$ & {$[64]$} \\
\hline
\end{tabular}

$\mathrm{Xe}=$ xenon; $\mathrm{MH}=$ metal halide; $(\mathrm{HI}) \mathrm{LED}=$ (high intensity) light emitting diode; $\mathrm{PR}=$ photoresisitor; $\mathrm{PD}=$ photodiode $; \mathrm{EPC}=$ electronic photon counter; $\mathrm{CCD}, \mathrm{CID}$ and $\mathrm{CMOS}=$ charge-coupled device, charge-injection device and complementary-metal-oxide-semiconductor device; EBCCD = electron-bombarded CCD; GaAsP = Gallium Arsenide Phosphide); FLS and LSC = fluorescence and luminescence; QY = quantum yield; * LOD values are reported for particular assays described in the cited literature [50,57-62,64]. For RT-qPCR, LOD is given for amplified DNA product.

Incident photons strike the photocathode material, which is usually a thin vapor-deposited conducting layer on the inside of the entry window of the device. Electrons are ejected from the surface as a consequence of the photoelectric effect. These electrons are directed by the focusing electrode toward the electron multiplier, which then multiplies them by the process of secondary emission [63].

A remarkable example of a fluorometry instrument is the Roche Light cycler 480 [61]. Designed for PCR analysis of nucleic acids, this instrument has a highly sensitive and innovative detection system, which can also be applied to enzyme-free assays. The fundamental innovation in this system is the incorporation of a charge coupled device (CCD) as a detector instead of PMT. A CCD is an integrated circuit etched onto a silicon surface forming light sensitive elements called pixels [59]. Photons incident on this surface generate charge that can be read by electronics and turned into a digital copy of the obtained light signal. CCDs come in a wide variety of types and are used in a range of applications from cell phone cameras to advanced fluorescence microscopes.

Optical microscopy has the most advanced equipment among the optical detection methods listed in Table 1. Using a suitable lamp, laser, or versatile semiconductor light source (LEDs) with a low-light 
CCD detector, microscopy allows for the direct detection of single molecules in complex biological samples [20-23]. In fluorescence microscopy, the sensitivity is defined by multiple parameters such as quantum efficiency (QE), limiting spatial resolution, contrast, modulation transfer function (MTF), and the contrast transfer function (CTF) for the electronic detector. QE refers to the detector signal as a function of the wavelength of the incident light. Limiting spatial resolution indicates the minimum separation required for the discrimination between two high contrast objects. MTF and CTF demonstrate the magnitude of the detector response as a function of spatial frequency [62].

Several variations of the basic CCD concept are commercially available for the analysis of low-light samples. These devices include charge-injection devices (CID), complementary metal-oxidesemiconductor detectors (CMOS) and the recently introduced electron-bombarded CCDs (EBCCD). In the CID and CMOS detectors, each individual photosensor has an associated amplifier. These image intensifiers increase the sensitivity and resolution of CCDs in microscopy by up to $75 \%$ compared to that of the CCD alone. Additionally, these devices have a high QE due to the fact that the photon travels through an input photocathode. Afterwards the photon is captured by a micro-channel plate electron multiplier, and is finally exposed onto a phosphorescent output screen. Similarly in EBCCD devices, a photocathode detects the photons but does so more powerfully than in the intensified CCD. Moreover, an EBCCD usually has a much higher spatial resolution and better signal-to-noise ratio (S/N) at moderate light levels. However, it also has a limited gain adjustment range and a modest low-light detection capability. Finally, GaAsP (Gallium Arsenide Phosphide) is an alternative detector, which has an even higher QE than PMT and can be suitable for detection of low-abundance targets.

For measuring low-abundance targets in these fluorescent microscopy systems it is important that the electronic detectors are extremely sensitive because of problems with "photobleaching". This is the photodestruction of fluorophores upon excitation in the presence of oxygen. In a typical fluorescence experiment, only about 5\%-10\% of the emitted light from an excited fluorophore is collected and transferred to the detector [63]. There are two approaches to detecting as much of the light from the probe as possible: (1) integration and image intensification with a slow-scanning video rate and (2) a progressive-scan CCD. Generally, a progressive-scan CCD produces a higher $\mathrm{S} / \mathrm{N}$ than an intensified slow-scanning analogue, provided sufficient integration time has been applied. Therefore slowscanning CCDs dominate most applications in the current research on low-abundance molecules [63].

Lastly, SPR techniques offer great sensitivity without a need for labeling the biomolecules. With this the sensing mechanism is rather different than in fluorometry or fluorescence microscopy therefore a different instrument design has to be applied. Four main SPR instrument configurations are available to date including prism-coupled total reflection systems, optical fibers, grating-coupled systems, and optical waveguide systems [64]. In these configurations, signal enhancing is typically achieved using the polymers and metal surface-coating techniques that we described previously. In more advanced SPR instruments the detector is usually a photodiode or CCD camera.

\section{Conclusion and Perspective: Moving toward Sensitive, Specific and Detailed Nucleic Acid Analysis Using Non-PCR Assays}

In conclusion, the signal-enhancing strategies described in this review enable sensitive analysis of nucleic acid targets at low concentrations without a need for target amplification. This is important because it minimizes interference with the initial sample composition. Generally, the signal-enhancing 
strategies we described can be classified as either enzyme-based or enzyme-free. Both groups rely on the specific binding of the target by probes, followed by signal enhancing and finally, detection. Attempts to increase the target-associated signal are pursued in parallel with attempts to decrease the off-target background signals. Thus, specificity of the probe hybridization to the target is crucial for all the assays described [65]. Promising strategies, which increase the specificity without amplifying the target, include target pre-enrichment using bait probes, modified nucleotide chemistries such as LNA and advanced rational probe designs [65-67]. Highly specific LNA/DNA probes also enable precise detection of SNPs and SNVs [66]. Additionally, with high levels of specificity, low abundance targets such as circulating DNA can be concentrated by orders of magnitude with available kits and in that way allow for the rapid analysis of e.g., clinical samples [67].

In this paper we also discussed promising new labels for optical assays including macrofluorophores, gold nanoparticles and other biopolymers, modified nucleic acid analogues, and nucleic acid binding dyes. As these fluorescent ultra-bright probes for PCR-independent nucleic acid detection develop, they might benefit from new chemical substances, nanotechnology and straightforward biotechnological approaches to enhance the signal [68-70]. Among other methods, fluorescence microscopy and SPR are two very promising techniques with a high potential for future biomolecular science and diagnostics. Furthermore, by using the highly sensitive detectors described above, one can detect low abundance nucleic acids at a single-molecule level, directly in cells and tissues, and in SPR methods without the need for additional labeling. This opens up an exciting opportunity for new insight into biomolecular interactions, cellular processes, and disease development. Amplification-free detection has the potential to create diagnostic tools and therapeutics while simultaneously testing them in the most biologically relevant fashion.

\section{Acknowledgments}

We acknowledge financial support from The Augustinus Foundation (Grant No. 95-305-73949).

\section{Conflicts of Interest}

The authors declare no conflict of interest.

\section{References}

1. Spengler, M.; Adler, M.; Niemeyer, C.M. Highly sensitive ligand-binding assays in pre-clinical and clinical applications: Immuno-PCR and other emerging techniques. Analyst 2015, doi:10.1039/C5AN00822K.

2. Jin, D.J.; Cagliero, C.; Martin, C.M.; Izard, J.; Zhou, Y.N. The dynamic nature and territory of transcriptional machinery in the bacterial chromosome. Front. Microbiol. 2015, 6, 1-14.

3. The Cancer Genome Atlas Research Network. Comprehensive genomic characterization of squamous cell lung cancers. Nature 2012, 489, 519-525.

4. Astakhova, K. Toward Non-Enzymatic Ultrasensitive Identification of Single Nucleotide Polymorphisms by Optical Methods. Chemosensors 2014, 2, 193-206. 
5. Hu, R.; Liu, T.; Zhang, X.B.; Yang, Y.; Chen, T.; Wu, C.; Liu, Y.; Zhu, G.; Huan, S.; Fu, T.; et al. DLISA: A DNAzyme-based ELISA for protein enzyme-free immunoassay of multiple analytes. Anal. Chem. 2015, 87, 7746-7753.

6. Song L.; Shan D.; Zhao M.; Pink B.A.; Minnehan K.A.; York L.; Gardel M.; Sullivan S.; Phillips A.F.; Hayman R.B.; Walt D.R.; Duffy D.C. Direct detection of bacterial genomic DNA at sub-femtomolar concentrations using single molecule arrays. Anal. Chem. 2013, 85, 1932-1939.

7. Wabuyele, M.B.; Farquar, H.; Stryjewski, W.; Hammer, R.P.; Soper, S.A.; Cheng, Y.W.; Barany, F. Approaching real-time molecular diagnostics: Single-pair fluorescence resonance energy transfer (spFRET) detection for the analysis of low abundant point mutations in K-ras oncogenes. J. Am. Chem. Soc. 2003, 125, 6937-6945.

8. Kosman, J.; Juskowiak, B. Peroxidase-mimicking DNAzymes for biosensing applications: A review. Anal. Chim. Acta 2011, 707, 7-17.

9. Zhang, M.; Li, H.; Deng, M.; Weng, X.; Ma, H.; Feng, S.; Zhou, Y.; Zhou, X. Studies of the activity of peroxidase-like DNAzyme by modifying 3'- or 5'-end of aptamers. Chem. Biodivers. 2012, 9, 170-180.

10. Kong, D.M.; Cai, L.L.; Guo, J.H.; Wu, J.; Shen, H.X. Characterization of the G-quadruplex structure of a catalytic DNA with peroxidase activity. Biopolymers 2009, 91, 331-339.

11. Spittle, C.; Ward, M.R.; Nathanson, K.L.; Gimotty, P.A.; Rappaport E.; Brose, M.S.; Medina A.; Letrero R.; Herlyn, M.; Edwards, R.H. Application of a BRAF pyrosequencing assay for mutation detection and copy number analysis in malignant melanoma. J. Mol. Diagn. 2007, 9, 464-471.

12. Song, Y.; Wang, X.; Zhao, C.; Qu, K.; Ren, J.; Qu, X. Label-free colorimetric detection of single nucleotide polymorphism by using single-walled carbon nanotube intrinsic peroxidase-like activity. Chem. Eur. J. 2010, 16, 3617-3621.

13. Dirks, R.M.; Pierce, N.A. Triggered amplification by hybridization chain reaction. Proc. Natl. Acad. Sci. USA 2004, 101, 15275-15278.

14. Porichis, F.; Hart, M.G.; Griesbeck, M.; Everett, H.L.; Hassan, M.; Baxter, A.E.; Lindqvist, M.; Miller, S.M.; Soghoian, D.Z.; Kavanagh, D.G.; et al. High-throughput detection of miRNAs and gene-specific mRNA at the single-cell level by flow cytometry. Nat. Commun. 2014, 5, 5641-5654.

15. Bishop, R. Applications of fluorescence in situ hybridization (FISH) in detecting genetic aberrations of medical significance. Biosci. Horiz. 2010, 3, 85-92.

16. Bystricky, K. Chromosome dynamics and folding in eukaryotes: Insights from live cell microscopy. FEBS Lett. 2015, doi:10.1016/j.febslet.2015.07.012.

17. Bowling, A.J.; Pence, H.E.; Church, J.B. Application of a novel and automated branched DNA in situ hybridization method for the rapid and sensitive localization of mRNA molecules in plant tissues. Appl. Plant Sci. 2014, 2, doi:10.3732/apps.1400011.

18. Baumeister, M.A.; Zhang, N.; Beas, H.; Brooks, J.R.; Canchola, J.A.; Cosenza, C.; Kleshik, F.; Rampersad, V.; Surtihadi, J.; Battersby, T. A sensitive branched DNA HIV-1 signal amplification viral load assay with single day turnaround. PLOS ONE 2012, 7, e33295.

19. Patel, J.A.; Anderson, E.; Dong, J. False Positive Ultrasensitive HIV bDNA Viral load results in diagnosis of perinatal HIV-Infection in the era of low transmission. Lab. Med. 2009, 40, 611-614. 
20. Briley, W.E.; Bondy, M.H.; Randeria, P.S.; Dupperc, T.J.; Mirkin, C.A. Quantification and real-time tracking of RNA in live cells using Sticky-flares. Proc. Natl. Acad. Sci. USA 2015, 112, 9591-9595.

21. Rino, J.; Martin, R.M.; Carvalho, C.; de Jesus, A.C.; Carmo-Fonseca, M. Single-Molecule Imaging of RNA Splicing in Live Cells. Method Enzymol. 2015, 558, 571-585.

22. Viswanathan, S.; Williams, M.E.; Bloss, E.B.; Stasevich, T.J.; Speer, C. M.; Nern, A.; Pfeiffer, B.D.; Hooks, B.M.; Li, W.P.; English, B.P.; et al. High-performance probes for light and electron microscopy. Nat. Methods 2015, 12, 568-576.

23. Gerling, T.; Wagenbauer, K.F.; Neuner, A.M.; Dietz, H. Dynamic DNA devices and assemblies formed by shape-complementary, non-base pairing 3D components. Science 2015, 347, 1446-1452.

24. Astakhova, I.K.; Samokhina, E.; Babu, B.R.; Wengel, J. Novel (Phenylethynyl)pyrene-LNA Constructs for Fluorescence SNP Sensing in Polymorphic Nucleic Acid Targets. ChemBioChem 2012, 13, 1509-1519.

25. Astakhova, I.K.; Korshun, V.A.; Jahn, K.; Kjems, J.; Wengel, J. Perylene Attached to 2'-Amino-LNA: Synthesis, Incorporation into Oligonucleotides, and Remarkable Fluorescence Properties in vitro and in Cell Culture. Bioconjug. Chem. 2008, 19, 1995-2007.

26. Okholm, A.; Kjemsa, J.; Astakhova, K. Fluorescence detection of natural RNA using rationally designed "clickable" oligonucleotide probes. RSC Adv. 2014, 4, 45653-45656.

27. Jørgensen, A.S.; Gupta, P.; Wengel, J.; Astakhova, I.K. "Clickable" LNA/DNA probes for fluorescence sensing of nucleic acids and autoimmune antibodies. Chem. Commun. 2013, 49, 10751-10753.

28. Dziuba, D.; Pohl, R.; Hocek, M. Bodipy-labeled nucleoside triphosphates for polymerase synthesis of fluorescent DNA. Bioconjug. Chem. 2014, 25, 1984-1995.

29. Yan, Z.; Gan, N.; Zhang, H.; Wang, D.; Qiao, L.; Cao, Y.; Li, T.; Hu, F. A sandwich-hybridization assay for simultaneous determination of HIV and tuberculosis DNA targets based on signal amplification by quantum dots-PowerVision $\left({ }^{\mathrm{TM}}\right)$ polymer coding nanotracers. Biosens. Bioelectron. 2015, 71, 207-213.

30. Yuan, Z.; Chen, Y.C.; Li, H.W.; Chang, H.T. Fluorescent silver nanoclusters stabilized by DNA scaffolds. Chem. Commun. 2014, 50, 9800-9815.

31. Li, J.; You, J.; Zhuang, Y.; Han, C.; Hu, J.; Wang, A.; Xu, K.; Zhu, J.J. A "light-up" and "spectrum-shift" response of aptamer-functionalized silver nanoclusters for intracellular mRNA imaging. Chem. Commun. 2014, 50, 7107-7110.

32. Dziuba, D.; Pohl, R.; Hocek, M. Polymerase synthesis of DNA labelled with benzylidene cyanoacetamide-based fluorescent molecular rotors: Fluorescent light-up probes for DNA-binding proteins. Chem. Commun. 2015, 51, 4880-4882.

33. Suzuki, A.; Saito, M.; Katoh, R.; Saito, Y. Synthesis of 8-aza-3,7-dideaza-2'-deoxyadenosines possessing a new adenosine skeleton as an environmentally sensitive fluorescent nucleoside for monitoring the DNA minor groove. Org. Biomol. Chem. 2015, 13, 7459-7468.

34. Kanamori, T.; Ohzeki, H.; Masaki, Y.; Ohkubo, A.; Takahashi, M.; Tsuda, K.; Ito, T.; Shirouzu, M.; Kuwasako, K.; Muto, Y.; et al. Controlling the fluorescence of benzofuran-modified uracil residues in oligonucleotides by triple-helix formation. ChemBioChem 2015, 16, 167-176. 
35. Hopkins, P.A.; Sinkeldam, R.W.; Tor, Y. Visibly emissive and responsive extended 6-aza-uridines. Org. Lett. 2014, 16, 5290-5293.

36. Riedl, J.; Ménová, P.; Pohl, R.; Orság, P.; Fojta, M.; Hocek, M. GFP-like fluorophores as DNA labels for studying DNA-protein interactions. J. Org. Chem. 2012, 77, 8287-8293.

37. Çamli, T.; Tuncel, M.; Şenel, S.; Tuncel, A. Functional, uniform and macroporous latex particles: Preparation, electron microscopic characterisation, and nonspecific protein adsorption properties. J. Appl. Polym. Sci. 2002, 84, 414-429.

38. Zhu, X.; Li, J.; He, H.; Huang, M.; Zhang, X.; Wang, S. Application of nanomaterials in the bioanalytical detection of disease-related genes. Biosens. Bioelectron. 2015, 74, 113-133.

39. Introduction to Signal Amplification-Section 6.1. Available online: https://www.life technologies.com/dk/en/home/references/molecular-probes-the-handbook/ultrasensitive-detectiontechnology/introduction-to-detection-methods.html (accessed on 5 August 2015).

40. Ihara, T.; Tanaka, S.; Chikaura, Y.; Jyo, A. Preparation of DNA-modified nanoparticles and preliminary study for colorimetric SNP analysis using their selective aggregations. Nucleic Acids Res. 2004, 32, e105.

41. Thomson, D.A.C.; Tee, E.H.-L.; Tran, N.T.D.; Monteiro, M.J.; Cooper, M.A. Oligonucleotide and polymer functionalized nanoparticles for amplification-free detection of DNA. Biomacromolecules 2012, 13, 1981-1989.

42. Mao, F.; Leung, W.Y.; Xin, X. Characterization of EvaGreen and the implication of its physicochemical properties for qPCR applications. BMC Biotechnol. 2007, 7, 76.

43. Nafisi, S.; Saboury, A.A.; Keramat, N.; Neault, J.F.; Tajmir-Riahi, H.A. Stability and structural features of DNA intercalation with ethidium bromide, acridine orange and methylene blue. J. Mol. Struct. 2007, 827, 35-43.

44. QuantiFluor® dsDNA System. Available online: https://dk.promega.com/products/dna-and-rnapurification/dna-and-rna-quantitation/sensitive-easy-to-use-quantifluor-dsdna-quantitation/quantifluordsdna-system/ (accessed on 5 August 2015).

45. Bhunia, S.K.; Saha, A.; Maity, A.R.; Ray, S.C.; Jana, N.R. Carbon nanoparticle-based fluorescent bioimaging probes. Sci. Rep. 2013, doi:10.1038/srep01473.

46. Misra, S.K.; Ohoka, A.; Kolmodin, N.J.; Pan, D. Next generation carbon nanoparticles for efficient gene therapy. Mol. Pharm. 2015, 12, 375-385.

47. Wang, L.; Cheng, Y.; Wang, H.; Li, Z. A homogeneous fluorescence sensing platform with water-soluble carbon nanoparticles for detection of microRNA and nuclease activity. Analyst 2012, 137, 3667-3672.

48. Mirkin, C.A.; Letsinger, R.L.; Mucic, R.C.; Storhoff, J.J. A DNA-based method for rationally assembling nanoparticles into macroscopic materials. Nature 1996, 382, 607-609.

49. Kang, J.H.; Asami, Y.; Murata, M.; Kitazaki, H.; Sadanaga, N.; Tokunaga, E.; Shiotani, S.; Okada, S.; Maehara, Y.; Niidome, T.; et al. Gold nanoparticle-based colorimetric assay for cancer diagnosis. Biosens. Bioelectron. 2010, 25, 1869-1874.

50. Liu, P.; Yang, X.; Sun, S.; Wang, Q.; Wang, K.; Huang, J.; Liu, J.; He, L. Enzyme-Free Colorimetric Detection of DNA by Using Gold Nanoparticles and Hybridization Chain Reaction Amplification. Anal. Chem. 2013, 85, 7689-7695. 
51. Su, X.; Teh, H.F.; Aung, K.M.; Zong, Y.; Gao, Z. Femtomol SPR detection of DNA-PNA hybridization with the assistance of DNA-guided polyaniline deposition. Biosens. Bioelectron. 2008, 23, 1715-1720.

52. Cao, D.; Huang, H.; Hu, M.; Cui, L.; Geng, F.; Rao, Z.; Niu, H.; Cai, Y.; Kang, Y. Comprehensive characterization of natural organic matter by MALDI- and ESI-Fourier transform ion cyclotron resonance mass spectrometry. Anal. Chim. Acta 2015, 866, 48-58.

53. Halpern, A.R.; Wood, J.B.; Wang, Y.; Corn, R.M. Previous Article Next Article Table of Contents Single-Nanoparticle Near-Infrared Surface Plasmon Resonance Microscopy for Real-Time Measurements of DNA Hybridization Adsorption. ACS Nano 2014, 8, 1022-1030.

54. Li, X.; Wang, Y.; Wang, L.; Wei, Q. A surface plasmon resonance assay coupled with a hybridization chain reaction for amplified detection of DNA and small molecules. Chem. Commun. 2014, 50, 5049-5052.

55. Qiu, X.; Liu, X.; Zhang, W.; Zhang, H.; Jiang, T.; Fan, D.; Luo, Y. Dynamic Monitoring of MicroRNA-DNA Hybridization Using DNAase-Triggered Signal Amplification. Anal. Chem. 2015, 87, 6303-6310.

56. Zagorodko, O.; Spadavecchia, J.; Serrano, A.Y.; Larroulet, I.; Pesquera, A.; Zurutuza, A.; Boukherroub, R.; Szunerits, S. Highly Sensitive Detection of DNA Hybridization on Commercialized Graphene-Coated Surface Plasmon Resonance Interfaces. Anal. Chem. 2014, 86, 11211-11216.

57. Konica Minolta. Available online: http://www.konicaminolta.com/instruments/ (accessed on 5 August 2015).

58. GloMax®-Multi Detection System. Available online: https://dk.promega.com/products/instruments/ multimode-readers/glomax_multi-detection-system/ (accessed on 5 August 2015).

59. Fluoromax-4. Available online: http://www.horiba.com/fileadmin/uploads/Scientific/Documents/ Fluorescence/Fluoromax-4.pdf (accessed on 5 August 2015).

60. TECAN. Available online: http://www.tecan.com/page/content/index.asp?MenuID=23\&ID= $10 \&$ Menu=1\&Item=21.2 (accessed on 5 August 2015).

61. LightCycler® 480 System. Available online: http://www.roche.com/products/productdetails.htm?type=product\&id=64 (accessed on 5 August 2015).

62. Murphy, D.B.; Davidson, M.W. Fundamentals of Light Microscopy and Electronic Imaging, 1st ed.; Wiley-Liss: New York, NY, USA, 2001.

63. Microscopy Resource Center. Available online: http://www.olympusmicro.com/primer/techniques/ fluorescence/fluorointrohome.html (accessed on 5 August 2015).

64. Homola, J. Surface plasmon resonance sensors for detection of chemical and biological species. Chem. Rev. 2008, 108, 462-493.

65. Zhang, D.Y.; Chen, S.X.; Yin, P. Optimizing the specificity of nucleic acid hybridization. Nat. Chem. 2012, 4, 208-214.

66. Wang, J.S.; Zhang, D.Y. Simulation-guided DNA probe design for consistently ultraspecific hybridization. Nat. Chem. 2015, 7, 545-553. 
67. Ramos, E.; Levinson, B.T.; Chasnoff, S.; Hughes, A.; Young, A.L.; Thornton, K.; Li, A.; Vallania, F.L.M.; Province, M.; Druley, T.E. Population-based rare variant detection via pooled exome or custom hybridization capture with or without individual indexing. BMC Genomics 2012, doi:10.1186/1471-2164-13-683.

68. Cui, M.; Fan, M.; Jing, R.; Wang, H.; Qin, J.; Sheng, H.; Wang, Y.; Wu, X.; Zhang, L.; Zhu, J.; et al. Cell-Free Circulating DNA: A New Biomarker for the Acute Coronary Syndrome. Cardiology 2013, 124, 76-84.

69. Choi, N.W.; Kim, J.; Chapin, S.C.; Duong, T.; Donohue, E.; Pandey, P.; Broom, W.; Hill, W.A.; Doyle, P.S. Multiplexed Detection of mRNA Using Porosity-Tuned Hydrogel Microparticles. Anal. Chem. 2012, 84, 9370-9378.

70. Blin, A.; Cissé, I.; Bockelmann, U. Electronic hybridization detection in microarray format and DNA genotyping. Sci. Rep. 2014, doi:10.1038/srep04194.

(C) 2015 by the authors; licensee MDPI, Basel, Switzerland. This article is an open access article distributed under the terms and conditions of the Creative Commons Attribution license (http://creativecommons.org/licenses/by/4.0/). 